\title{
Association of NAD(P)H Quinine Oxidoreductase 1 rs1800566 Polymorphism with Bladder and Prostate Cancers - a Systematic Review and Meta-Analysis
}

\author{
Asociace polymorfizmu NAD(P)H chininové oxidoreduktázy 1 \\ rs1800566 s karcinomem močového měchýře a prostaty - \\ systematický přehled a metaanalýza
}

\author{
Abedinzadeh M. ${ }^{1}$, Moghimi M. ${ }^{2}$, Dastgheib S. A. ${ }^{3}$, Maleki H. ${ }^{1}$, Salehi E. ${ }^{4}$, Mohammad Z. ${ }^{5}$, \\ Jarahzadeh M. H. ${ }^{6}$, Neamatzadeh $\mathrm{H}^{7}{ }^{7,8}$
}

\begin{abstract}
Summary
Background: Number of studies has been performed to investigate the association of $\mathrm{NAD}(\mathrm{P}) \mathrm{H}$ quinine oxidoreductase 1 (NQO1) rs 1800566 polymorphism with risk of bladder and prostate cancers, but presented inconsistent results. Therefore, we performed a meta-analysis to provide a comprehensive data on the association of NQO1 rs 1800566 polymorphism with bladder and prostate cancers. Methods: All eligible studies were identified in PubMed, Google Scholar, EMBASE, and China National Knowledge Infrastructure databases before June 01, 2019. Results: A total of 22 case-control studies including 15 studies with 4,413 cases and 4,275 controls on bladder cancer and 7 studies with 762 cases and 1,813 controls on prostate cancer were selected. Overall, pooled data showed that the NQO1 rs1800566 polymorphism was significantly associated with an increased risk of bladder cancer (T vs. C: OR $1.300 ; 95 \% \mathrm{CI}$ $1.112-1.518 ; \mathrm{P}=0.001 ; \mathrm{TT}$ vs. $\mathrm{CC}: \mathrm{OR} 1.415 ; 95 \% \mathrm{Cl} 1.084-1.847 ; \mathrm{P}=0.011 ; \mathrm{TC}$ vs. CC: $\mathrm{OR} 1.389$; $95 \% \mathrm{Cl} 1.111-1.738 ; \mathrm{P}=0.004 ; \mathrm{TT}+\mathrm{TC}$ vs. CC: $\mathrm{OR} 1.428 ; 95 \% \mathrm{Cl} 1.145-1.782 ; \mathrm{P}=0.002)$ and prostate cancer (TC vs. CC: OR 1.276; $95 \% \mathrm{Cl} 1.047-1.555 ; \mathrm{P}=0.016 ; \mathrm{TT}+\mathrm{TC}$ vs. CC: OR $1.268 ; 95 \% \mathrm{Cl}$ $1.050-1.532 ; \mathrm{P}=0.014)$. The stratified analysis by ethnicity revealed an increased risk of bladder cancer among Caucasians and prostate cancer among Asians. Conclusion: This meta-analysis suggested that the NQO1 rs1800566 polymorphism was significantly associated with increased risk of bladder and prostate cancers.
\end{abstract}

Key words

urinary bladder neoplasms - prostatic neoplasms - NQO1 gene - polymorphism meta-analysis

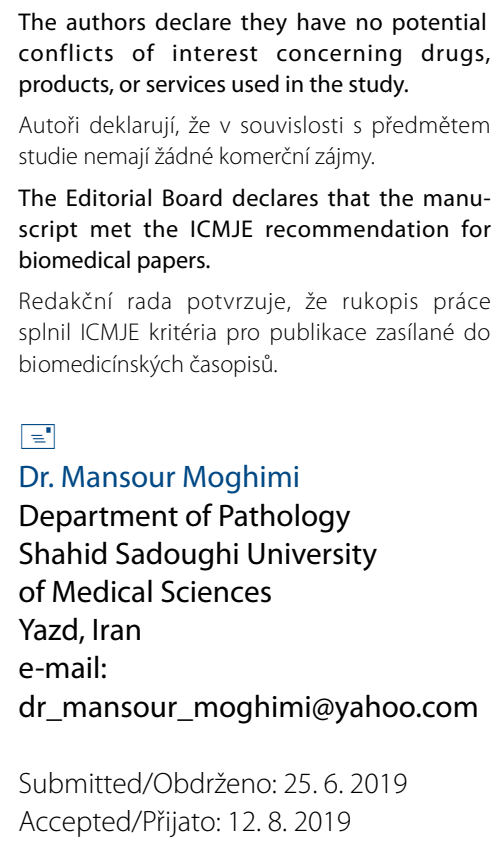

The authors declare they have no potential conflicts of interest concerning drugs, products, or services used in the study.

Autor̆i deklarují, že v souvislosti s předmětem studie nemaji žádné komerční zájmy.

The Editorial Board declares that the manuscript met the ICMJE recommendation for biomedical papers.

Redakční rada potvrzuje, že rukopis práce splnil ICMJE kritéria pro publikace zasilané do biomedicínských časopisů.

Dr. Mansour Moghimi Department of Pathology Shahid Sadoughi University of Medical Sciences Yazd, Iran e-mail: dr_mansour_moghimi@yahoo.com

Submitted/Obdrženo: 25. 6.2019 Accepted/Prijato: 12. 8. 2019

doi: 10.14735/amko202092 


\begin{abstract}
Souhrn
Východiska: Bylo provedeno několik studií s cílem zkoumání asociace polymorfizmu NAD(P)H chinin oxidoreduktázy 1 (NQO1) rs1800566 s rizikem karcinomu močového měchýře a prostaty, ale byly předloženy nekonzistentní výsledky. Proto jsme provedli metaanalýzu, abychom poskytli komplexní údaje o asociaci polymorfizmu NQO1 rs1800566 s karcinomem močového měchýře a prostaty. Metody: Př́slušné studie byly identifikovány v databázích PubMed, Google Scholar, EMBASE a China National Knowledge Infrastructure před 1. červnem 2019. Výsledky: Bylo vybráno celkem 22 prípadových kontrolních studií zahrnujících 15 studií karcinomu močového měchýře se 4413 prípady a 4275 kontrolami a 7 studií karcinomu prostaty s 762 prípady a 1813 kontrolami. Souhrnná data ukázala, že polymorfizmus NQO1 rs1800566 byl významně asociován se zvýšeným rizikem karcinomu močového měchýre (T vs. C: OR 1,300; $95 \% \mathrm{Cl} 1,112-1,518 ; p=0,001$; TT vs. CC: OR 1,415; 95\% Cl 1,084-1,847; $\mathrm{p}=0,011 ; \mathrm{TC}$ vs. CC: OR 1,389; $95 \% \mathrm{Cl} 1,111-1,738 ; \mathrm{p}=0,004 ; \mathrm{TT}+\mathrm{TC}$ vs. CC: OR 1,428; 95\% Cl 1,145-1,782; $\mathrm{p}=0,002)$ a karcinomu prostaty (TC vs. CC: OR 1,276; $95 \%$ Cl 1,047-1,555; $p=0,016 ; T T+$ TC vs. CC: OR 1,268; 95\% Cl 1,050-1,532; $p=0,014)$. Analýza stratifikovaná podle etnicity odhalila zvýšené riziko karcinomu močového měchýře u Kavkazanů a karcinomu prostaty u Asiatů. Závěr: Tato metaanalýza naznačuje, že polymorfizmus NQO1 rs1800566 byl významně spojen se zvýšeným rizikem karcinomu močového měchýre a prostaty.
\end{abstract}

Klíčová slova

karcinom močového měchýře - karcinom prostaty - gen NQO1 - polymorfizmus - metaanalýza

\section{Introduction}

Bladder cancer is one of the most common malignancies, with approximately 430,000 new cases diagnosed worldwide, with 118,000 new cases and 52,000 deaths recorded in Europe in 2012 [1]. Bladder cancer is a heterogeneous disease appearing in different forms, e.g. non-muscle invasive and muscle invasive. It mainly affects elderly people and the average age at the time of diagnosis is 73 years [2]. The leading risk factor for bladder cancer is tobacco use, with cigarette smokers showing an approximately threefold higher risk compared to non-smokers $[1,3]$. Moreover, prostate cancer is the most commonly diagnosed cancer and the second leading cause of cancer deaths among men in the United States [4]. Risk factors for developing localized prostate cancer are not well known. However, a family history of prostate cancer and age has long been identified as an important risk for development of the disease $[5,6]$. Although several notable advances in our knowledge about risk factors of bladder and prostate cancers were published in recent years, their exact mechanisms remains poorly understood [7]. It is suggested that both bladder and prostate cancers are caused by a combination of genetic and environmental risk factors [5].

The $\mathrm{NAD}(\mathrm{P}) \mathrm{H}$ quinine oxidoreductase 1 (NQO1; also known as diphtheria toxin diaphorase), a key phase II enzyme, plays an important role in the metabolism of several carcinogens; it also protects cells against oxidative stress $[8,9]$. NQO1 ac- tivity prevents the one electron reduction of quinones and thus prevents generation of free radicals by redox cycle. The human NQO1 gene is located on chromosome $16 q 22$, consists of seven exons (first exon is non-coding) spanning $20 \mathrm{~kb}$ of genomic DNA [10]. NQO1 genetic variations may play important roles in etiology of genitourinary malignancies, especially bladder cancer and prostate cancer. Therefore, NQO1 is considered as an anticancer enzyme, and its polymorphisms can highly impact bioreductive cancer therapy $[11,12]$.

Several single nucleotide polymorphisms have been identified in the NQO1 gene; among them, rs1800566 C>T (Pro187Ser) polymorphism at nucleotide 609 of exon 6, codes for a proline (Pro)-to-serine (Ser) amino acid substitution is one of the most studied [13]. Genotype-phenotype correlation studies have showen that the rs 1800566 polymorphism is associated with a decreased activity of NQO1 enzymatic activity and an increased susceptibility to carcinogenesis, xenobiotic induced toxicity and also a phenotypic gene-dose effect [14]. A number of case-control studies showed that the NQO1 rs1800566 polymorphism might increase susceptibility to bladder and prostate cancers, but the results remain inconclusive and contradictive. In addition, the sample size in each study was relatively small, and the statistical power might be insufficient. Therefore, we performed a comprehensive meta-analysis to derive a more precise estimate for association of the NQO1 rs1800566 poly- morphism with susceptibility to bladder and prostate cancers.

\section{Materials and methods \\ Publication search}

A comprehensive literature search was performed in PubMed, EMBASE, Cochrane Library database, Springer Link, Chinese Biomedical Database, China National Knowledge Infrastructure platforms, WanFang and VIP database to collect all the eligible studies evaluating the association of NQO1 rs1800566 polymorphism with bladder and prostate cancers up to June 01, 2019. The following terms, keywords and their combinations were used: ("Prostate cancer" or "Bladder cancer") and ("NAD(P)H dehydrogenase (Quinone) 1" or "NQO1" or "DT-diaphorase" or "DTD" or "quinone reductase") and ("609C>T" or "rs1800566" or "Pro187Ser") and ("Polymorphism" or "SNPs" or "Mutation" or "Variation" or "Allele"). Additionally, we reviewed the reference list of all relevant articles and reviews to identify potential eligible studies. If there were multiple publications from the same population, only the most recent was included.

\section{Selection criteria}

The eligible studies, included in the current meta-analysis, must have met the following criteria: 1) studies with case-control or cohort design; 2) studies focused on the association of NQO1 rs1800566 polymorphism with bladder and prostate cancers; 3 ) providing complete data of cases and controls for calculating an odds ratio (OR) with 
95\% confidence interval (CI). Studies were excluded for following reasons: 1) abstracts, reviews, case reports, posters, editorials, conference articles; 2) data unavailable for calculating genotype or allele frequencies; 3) studies without reported genotype frequencies; 4) case only studies (without controls); 5) linkage studies, twin and family-based studies; and 6) overlapping data or duplicate of previous publication.

\section{Data extraction}

Data were independently extracted by two authors (S. A. Dastgheib and H. Neamatzadeh) using a data-collecting form according to the inclusion criteria. Any disagreement was resolved by discussion with third author (M. Abedinzadeh). The following information was collected from each study: first author's name, year of publication, ethnicity, country of the selected subjects, source of the control groups, definition of metabolic syndrome, frequencies of genotypes in both groups and genotyping methods. Diverse ethnicity descents were categorized as Asian, Caucasian and African. If data were not reported in the primary manuscripts, we contacted the corresponding authors by email to request the missing data.

\section{Statistical analysis}

The strength of association between NQO1 polymorphism and bladder and prostate cancers was assessed by ORs with $95 \% \mathrm{Cls}$. The significance of the pooled effect size was determined by Z-test, in which $\mathrm{P}<0.05$ was considered statistically significant. The association was evaluated under all five genetic models, i.e., allele (T vs. C), homozygote (TT vs. CC), heterozygote (TC vs. CC), dominant (TT + TC vs. CC), and the recessive (TT vs. TC + CC). Between-study heterogeneity was evaluated by the Cochran Q-test, in which $\mathrm{P} \leq 0.10$ indicated significant heterogeneity was found. In addition, the $I^{2}$ statistic we applied to qualify between-study heterogeneity (range of $0-100 \%: I^{2}=0-25 \%$, no heterogeneity; $I^{2}=25-50 \%$, moderate heterogeneity; $\left.\right|^{2}=50-75 \%$, large heterogeneity; $I^{2}=75-100 \%$, extreme heterogeneity). The random effects model shows more

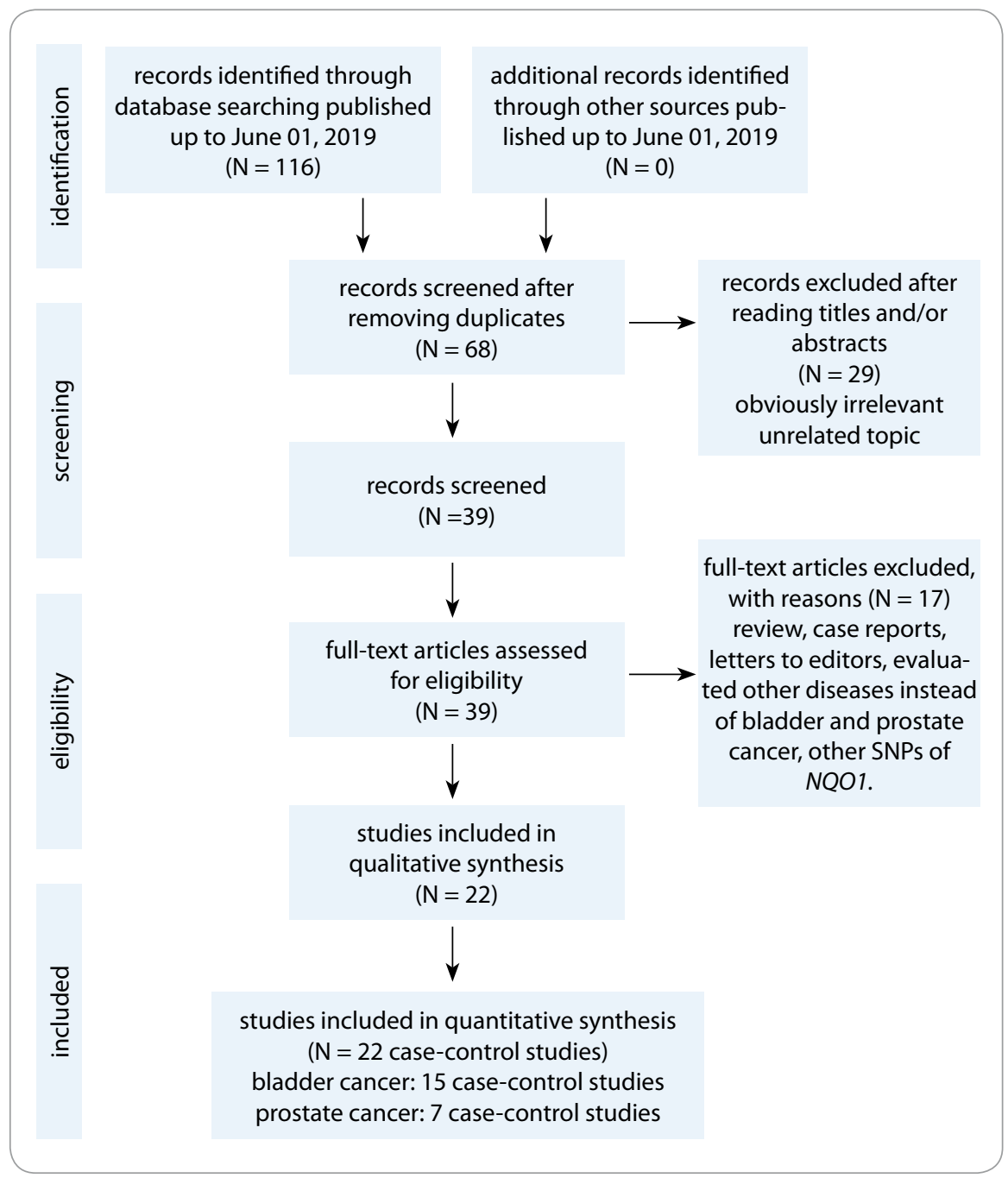

Fig. 1. Flowchart of literature search and selection process [43].

flexibility with respect to variable effect size in different studies and study populations. Thus, we have applied a random-effects model, using the DerSimonian and Laird method to calculate the pooled OR when heterogeneity was found; otherwise, affixed effect model was applied to use the Mantel-Haenszel method in absence of heterogeneity. A Hardy-Weinberg equilibrium (HWE) test of the NQO1 rs1800566 polymorphism in controls was tested using chisquare test $(P$-values $<0.05)$. Subgroup analyses were conducted by stratification of ethnicity to identifying potential source of heterogeneity. Sensitivity analyses were performed to assess influence of each single study on pooled ORs and the stability of the meta-analysis results by sequential removal of individual studies. In addition, sensitivity analysis was performed to examine the stability of the results by excluding those studies that did not show HWE. Funnel plots and Egger's linear regression test were used to estimate evidence for potential publication bias. All of the statistical calculations were performed using Comprehensive Meta-Analysis software version 2.0 (Biostat, USA). Two-sided P-values $<0.05$ were considered statistically significant.

\section{Results \\ Study characteristics}

Fig. 1 shows the flowchart of literature search and selection process. The initial literature searches retrieved 116 potentially relevant studies. After reading the titles and abstracts, 48 studies were 
Tab. 1. Characteristics of studies included in the meta-analysis.

\begin{tabular}{|c|c|c|c|c|c|c|c|c|c|c|c|c|c|c|c|c|}
\hline \multirow[t]{3}{*}{ First Author } & \multirow{3}{*}{$\begin{array}{c}\text { Country } \\
\text { (Ethnicity) }\end{array}$} & \multirow{3}{*}{$\begin{array}{l}\text { Geno- } \\
\text { typing } \\
\text { Method }\end{array}$} & \multirow[t]{3}{*}{ SOC } & \multirow{3}{*}{$\begin{array}{l}\text { Case/ } \\
\text { Control }\end{array}$} & \multicolumn{5}{|c|}{ Cases } & \multicolumn{5}{|c|}{ Controls } & \multirow[t]{3}{*}{ MAFs } & \multirow[t]{3}{*}{ HWE } \\
\hline & & & & & \multicolumn{3}{|c|}{ Genotypes } & \multicolumn{2}{|c|}{ Allele } & \multicolumn{3}{|c|}{ Genotypes } & \multicolumn{2}{|c|}{ Allele } & & \\
\hline & & & & & CC & CT & TT & C & $\mathbf{T}$ & CC & CT & TT & C & $\mathbf{T}$ & & \\
\hline \multicolumn{17}{|l|}{ Bladder Cancer } \\
\hline Schulz 1996 [15] & $\begin{array}{l}\text { Germany } \\
\text { (Caucasian) }\end{array}$ & PCR-RFLP & PB & $99 / 260$ & 68 & 26 & 5 & 162 & 36 & 195 & 61 & 4 & 451 & 69 & 0.13 & 0.75 \\
\hline Park 2003 [21] & $\begin{array}{c}\text { USA } \\
\text { (Caucasian) }\end{array}$ & CR-RFLP & $\mathrm{HB}$ & $232 / 239$ & 142 & 82 & 8 & 366 & 96 & 163 & 66 & 10 & 392 & 86 & 0.18 & 0.321 \\
\hline Choi 2003 [22] & Korea (Asian) & PCR-RFLP & $\mathrm{HB}$ & $177 / 170$ & 81 & 68 & 28 & 230 & 124 & 94 & 60 & 16 & 248 & 92 & 0.27 & 0.16 \\
\hline Sanyal 2004 [23] & $\begin{array}{c}\text { Sweden } \\
\text { (Caucasian) }\end{array}$ & CR-RFLP & PB & $299 / 124$ & 206 & 85 & 8 & 497 & 101 & 83 & 34 & 7 & 200 & 48 & 0.19 & 0.175 \\
\hline Hung 2004 [24] & $\begin{array}{c}\text { Italy } \\
\text { (Caucasian) }\end{array}$ & PCR-RFLP & HB & $201 / 214$ & 113 & 75 & 13 & 301 & 101 & 135 & 66 & 13 & 336 & 92 & 0.21 & 0.207 \\
\hline Moore 2004 [25] & $\begin{array}{l}\text { Argentina } \\
\text { (Caucasian) }\end{array}$ & PCR-RFLP & PB & $106 / 108$ & 62 & 35 & 9 & 157 & 53 & 61 & 40 & 7 & 162 & 54 & 0.25 & 0.89 \\
\hline Terry 2005 [26] & $\begin{array}{c}\text { USA } \\
\text { (Caucasian) }\end{array}$ & MS & HB & $235 / 214$ & 156 & 70 & 9 & 382 & 88 & 150 & 58 & 6 & 358 & 70 & 0.16 & 0.891 \\
\hline Broberg 2005 [27] & $\begin{array}{l}\text { Sweden } \\
\text { (Caucasian) }\end{array}$ & MS & PB & $179 / 156$ & 43 & 131 & 5 & 217 & 141 & 107 & 46 & 3 & 260 & 52 & 0.17 & 0.44 \\
\hline Wang 2007 [19] & Taiwan (Asian) & PCR-RFLP & $\mathrm{HB}$ & $300 / 300$ & 70 & 148 & 82 & 288 & 312 & 94 & 136 & 70 & 324 & 276 & 0.46 & 0.12 \\
\hline $\begin{array}{l}\text { Figueroa } 2008 \\
\text { [28] }\end{array}$ & $\begin{array}{c}\text { Spain } \\
\text { (Caucasian) }\end{array}$ & qMan & $\mathrm{HB}$ & $1128 / 1123$ & 685 & 392 & 51 & 1762 & 494 & 661 & 400 & 62 & 1722 & 524 & 0.23 & 884 \\
\hline $\begin{array}{l}\text { Pandith } 2011 \\
\text { [16] }\end{array}$ & $\begin{array}{l}\text { India } \\
\text { (Asian) }\end{array}$ & PCR-RFLP & HB & $104 / 120$ & 44 & 53 & 7 & 141 & 67 & 70 & 44 & 6 & 184 & 56 & 0.23 & 0.785 \\
\hline Fu 2003 [20] & China (Asian) & PCR-RFLP & $\mathrm{HB}$ & $9 / 100$ & 30 & 38 & 31 & 68 & 100 & 38 & 46 & 16 & 122 & 78 & 0.39 & 0.739 \\
\hline Huang 2014 [8] & Taiwan (Asian) & PCR-RFLP & $\mathrm{HB}$ & $159 / 150$ & 36 & 83 & 40 & 155 & 163 & 51 & 67 & 32 & 169 & 131 & 0.44 & 0.259 \\
\hline $\begin{array}{l}\text { Goerlitz } 2014 \\
\text { [17] }\end{array}$ & $\begin{array}{l}\text { Egypt } \\
\text { (African) }\end{array}$ & aqMan & PB & $895 / 797$ & 519 & 323 & 53 & 1361 & 429 & 470 & 276 & 51 & 1216 & 378 & 0.24 & 0.226 \\
\hline Mandal 2012 [18] & India (Asian) & PCR-RFLP & $\mathrm{HB}$ & $200 / 200$ & 105 & 72 & 23 & 282 & 118 & 128 & 61 & 11 & 317 & 83 & 0.21 & 0.304 \\
\hline \multicolumn{17}{|l|}{ Prostate Cancer } \\
\hline Steiner 1999 [29] & $\begin{array}{l}\text { Germany } \\
\text { (Caucasian) }\end{array}$ & PCR-RFLP & PB & 4/100 & 37 & 15 & 2 & 89 & 19 & 67 & 31 & 2 & 165 & 35 & 0.18 & 0.46 \\
\hline $\begin{array}{l}\text { Hamajima } 2002 \\
\text { [30] }\end{array}$ & $\begin{array}{l}\text { Japan } \\
\text { (Asian) }\end{array}$ & CR-RFLP & $\mathrm{HB}$ & $6 / 640$ & 17 & 30 & 9 & 64 & 48 & 240 & 286 & 114 & 766 & 514 & 0.40 & 0.075 \\
\hline Ergen 2007 [31] & $\begin{array}{c}\text { Turkey } \\
\text { (Caucasian) }\end{array}$ & PCR-RFLP & $\mathrm{HB}$ & $45 / 59$ & 23 & 17 & 5 & 63 & 27 & 23 & 26 & 10 & 72 & 46 & 0.39 & 0.571 \\
\hline $\begin{array}{l}\text { Steinbrecher } \\
2010[34]\end{array}$ & $\begin{array}{l}\text { Germany } \\
\text { (Caucasian) }\end{array}$ & MS & PB & $248 / 492$ & 163 & 80 & 5 & 406 & 90 & 333 & 133 & 26 & 799 & 185 & 0.19 & 0.011 \\
\hline $\begin{array}{l}\text { Jing-Xian } 2011 \\
\text { [33] }\end{array}$ & $\begin{array}{l}\text { China } \\
\text { (Asian) }\end{array}$ & aqMan & NS & $45 / 40$ & 5 & 26 & 14 & 36 & 54 & 12 & 21 & 7 & 36 & 35 & 0.44 & 0.673 \\
\hline Mandal 2012 [18] & India (Asian) & PCR-RFLP & $\mathrm{HB}$ & $195 / 250$ & 105 & 67 & 23 & 277 & 113 & 164 & 72 & 14 & 400 & 100 & 0.20 & 0.113 \\
\hline Stoehr 2012 [32] & $\begin{array}{l}\text { Germany } \\
\text { (Caucasian) }\end{array}$ & PCR-RFLP & HB & $119 / 232$ & 76 & 37 & 6 & 189 & 49 & 166 & 60 & 6 & 392 & 72 & 0.16 & .835 \\
\hline
\end{tabular}

SOC - source of control, MAF - minor allele frequency, HWE - Hardy-Weinberg equilibrium, PCR-RFLP - polymorphism chain reaction-restriction fragment length polymorphism, MS - mass spectrometry, PB - population based, HB - hospital based, NS - not stated 
A

Study name

\begin{tabular}{|c|c|c|c|c|c|}
\hline & tio & limit & limit & Z-Value & Val \\
\hline hulz 1996 [15] & 1.452 & 0.934 & 2.258 & 1.658 & 0.097 \\
\hline Park 2003 [21] & 1.189 & 0.860 & 1.644 & 1.048 & 0.295 \\
\hline Choi 2003 [22] & 1.453 & 1.051 & 2.009 & 2.262 & 0.024 \\
\hline Sanyal 2004 [23] & 0.847 & 0.579 & 1.239 & -0.856 & 0.392 \\
\hline Hung 2004 [24] & 1.225 & 0.888 & 1.692 & 1.236 & 0.217 \\
\hline Moore 2004 [25] & 1.000 & 0.646 & 1.549 & 0.000 & 1.000 \\
\hline Terry 2005 [26] & 1.178 & 0.834 & 1.664 & 0.930 & 0.352 \\
\hline Broberg 2005 [27] & 3.249 & 2.254 & 4.682 & 6.318 & 0.000 \\
\hline Wang 2008 [19] & 1.272 & 1.014 & 1.595 & 2.078 & 0.038 \\
\hline Figueroa 2008 [28] & 0.921 & 0.801 & 1.059 & -1.149 & 0.250 \\
\hline Pandith 2011 [16] & 1.561 & 1.029 & 2.370 & 2.093 & 0.036 \\
\hline Fu 2013 [20] & 1.596 & 1.072 & 2.376 & 2.303 & 0.021 \\
\hline Huang 2014 [8] & 1.357 & 0.988 & 1.862 & 1.887 & 0.059 \\
\hline Goerlitz 2014 [17] & 1.014 & 0.865 & 1.188 & 0.172 & 0.863 \\
\hline Mandal 2014 [18] & 1.598 & 1.157 & 2.208 & 2.842 & 0.004 \\
\hline & & & 1.51 & & \\
\hline
\end{tabular}

\section{Odds ratio and $95 \% \mathrm{Cl}$}

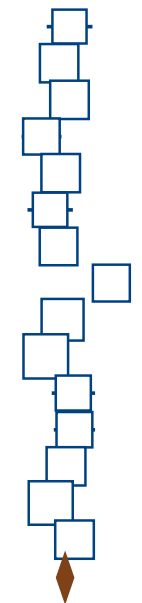

Relative weight

5.38

6.72

6.72

6.04

6.73

5.42

6.46

6.22

7.88

8.80

5.64

5.85

6.80

8.62

6.72

$\begin{array}{lllll}0.01 & 0.1 & 1 & 10 & 100\end{array}$

\section{Odds ratio and $95 \% \mathrm{Cl}$}

\section{Study name}

B

Steiner 1999 [29]

Hamajima 2002 [30]

Ergen 2007 [31]

Steinbrecher 2010 [34]

Jiang-xian 2011 [33]

Mandal 2012 [18]

Stoehr 2012 [32]
Statistics for each study

\section{Odds
ratio}

0.933

1.376

0.611

1.092

3.429

1.635

1.423

1.268

Lowe
limit
0.459
0.762
0.279
0.791
1.086
1.113
0.889
1.050

\section{Uimit}

1.897

2.487

1.339

1.509

10.824

2.399

2.278

1.532
Z-Value

$-0.192$

1.058

$-1.230$

0.535

2.101

2.509

1.470

2.470

\section{p-Value}

0.848

0.290

0.219

0.593

0.036

0.012

0.142

0.014
Relative weight

7.07

10.16

5.78

34.06

2.69

24.15

16.08

$\begin{array}{lllll}0.01 & 0.1 & 1 & 10 & 100\end{array}$

Fig. 2. Forest plot for association of NQO1 rs 1800566 polymorphism with risk of bladder and prostate cancers.

A. Bladder cancer (allele model T vs. C).

B. Prostate cancer (dominant model TT + TC vs. CC).

excluded. Among these studies, 46 studies were excluded because they did not report useful data for meta-analysis, or were a review, case only study, and not being case-control studies. Finally, 22 case-control studies including 15 studies with 4,413 cases and 4,275 controls for bladder cancer $[8,15-28]$ and 7 studies with 762 cases and 1,813 controls for prostate cancer [18,29-34] were included to the meta-analysis. The main characteristics of the studies are shown in Tab. 1. All included studies were published between 2005 and 2013. The studies have been carried out in Germany $(N=4)$, USA $(N=2)$, Sweden $(N=2)$,
India ( $\mathrm{N}=3)$, China $(\mathrm{N}=2)$, Taiwan $(\mathrm{N}=2)$, Korea $(\mathrm{N}=1)$, Italy $(\mathrm{N}=1)$, Argentina $(\mathrm{N}=1)$, Spain $(\mathrm{N}=1)$, Egypt $(\mathrm{N}=1)$, Japan $(\mathrm{N}=1)$, and Turkey $(\mathrm{N}=1)$. As for ethnicity, 12 studies were conducted on Caucasians, 9 studies on Asians, and 1 article on Africans. A total of 16 polymerase chain reaction-restriction fragment length polymorphism, 3 mass spectrometry, and 1 TaqMan genotyping approach were used. The genotype and minor allele frequency distributions in the studies considered in the present meta-analysis are shown in Tab. 1. Moreover, the distribution of genotypes in the controls was in agreement with HWE for all selected studies, except for one study for prostate cancer (Tab. 1).

\section{Quantitative synthesis \\ Bladder cancer}

The summary of the meta-analysis of the association of NQO1 rs1800566 polymorphism with bladder cancer is shown in Tab. 2. Overall, pooled ORs showed that there was a significant association between NQO1 rs1800566 polymorphism and bladder cancer risk under four genetic models, i.e., allele ( $T$ vs. C: OR 1.300, 95\% Cl 1.112-1.518, P = 0.001) (Fig. 2A), homozygote (TT vs. CC: OR $1.415,95 \% \mathrm{Cl} 1.084-1.847, \mathrm{P}=0.011$ ), 
Tab. 2. Summary of meta-analysis for the association of NQO1 rs1800566 polymorphism with risk of bladder and prostate cancers.

\begin{tabular}{|c|c|c|c|c|c|c|c|c|c|c|}
\hline \multirow[t]{2}{*}{ Subgroup } & \multirow[t]{2}{*}{ Genetic model } & \multirow{2}{*}{$\begin{array}{l}\text { Type of } \\
\text { model }\end{array}$} & \multicolumn{2}{|c|}{ Heterogeneity } & \multicolumn{4}{|c|}{ Odds ratio } & \multicolumn{2}{|c|}{ Publication bias } \\
\hline & & & $I^{2}(\%)$ & $\mathbf{P}_{\mathrm{H}}$ & OR & $95 \% \mathrm{Cl}$ & Z-test & POR & $P_{\text {Beggs }}$ & $\mathbf{P}_{\text {Eggers }}$ \\
\hline \multicolumn{11}{|c|}{ Bladder Cancer } \\
\hline \multirow[t]{5}{*}{ Overall } & Tvs. C & random & 76.45 & $\leq 0.001$ & 1.300 & $1.112-1.518$ & 3.299 & 0.001 & 0.276 & 0.014 \\
\hline & TT vs. CC & random & 47.96 & 0.020 & 1.415 & $1.084-1.847$ & 2.555 & 0.011 & 0.198 & 0.081 \\
\hline & TC vs. CC & random & 79.68 & $\leq 0.001$ & 1.389 & $1.111-1.738$ & 2.879 & 0.004 & 0.620 & 0.064 \\
\hline & TT + TC vs. CC & random & 81.29 & $\leq 0.001$ & 1.428 & $1.145-1.782$ & 3.157 & 0.002 & 0.198 & 0.023 \\
\hline & TT vs. TC + CC & fixed & 31.43 & 0.117 & 1.169 & $0.987-1.519$ & 1.838 & 0.066 & 0.488 & 0.209 \\
\hline
\end{tabular}

\section{Ethnicity}

\begin{tabular}{|c|c|c|c|c|c|c|c|c|c|c|}
\hline \multirow[t]{5}{*}{ Caucasian } & T vs. C & random & 84.98 & $\leq 0.001$ & 1.283 & $0.963-1.709$ & 1.706 & 0.088 & 0.710 & 0.171 \\
\hline & TT vs. CC & fixed & 38.57 & 0.122 & 0.986 & $0.748-1.300$ & -0.098 & 0.922 & 0.018 & 0.084 \\
\hline & TC vs. CC & random & 88.49 & $\leq 0.001$ & 1.405 & $0.934-2.115$ & 1.630 & 0.103 & 1.000 & 0.257 \\
\hline & $\mathrm{TT}+\mathrm{TC}$ vs. CC & random & 88.88 & $\leq 0.001$ & 1.398 & $0.939-2.082$ & 1.648 & 0.099 & 1.000 & 0.205 \\
\hline & TT vs. TC + CC & fixed & 6.630 & 0.379 & 0.938 & $0.714-1.232$ & -0.459 & 0.646 & 0.173 & 0.178 \\
\hline \multirow[t]{5}{*}{ Asian } & T vs. C & fixed & 0.00 & 0.842 & 1.421 & $1.249-1.616$ & 5.357 & $\leq 0.001$ & 0.259 & 0.027 \\
\hline & TT vs. CC & fixed & 0.00 & 0.880 & 1.890 & $1.445-2.472$ & 4.648 & $\leq 0.001$ & 0.259 & 0.148 \\
\hline & TC vs. CC & fixed & 0.00 & 0.757 & 1.469 & $1.208-1.787$ & 3.857 & $\leq 0.001$ & 1.000 & 0.922 \\
\hline & $\mathrm{TT}+\mathrm{TC}$ vs. CC & fixed & 0.00 & 0.961 & 1.583 & $1.318-1.901$ & 4.919 & $\leq 0.001$ & 1.000 & 0.500 \\
\hline & TT vs. TC + CC & fixed & 0.00 & 0.443 & 1.498 & $1.183-1.897$ & 3.359 & 0.001 & 0.259 & 0.191 \\
\hline
\end{tabular}

\section{Prostate Cancer}

Overall

\begin{tabular}{|c|c|c|}
\hline T vs. C & random & 55.59 \\
\hline TT vs. CC & random & 62.56 \\
\hline TC vs. CC & fixed & 1.030 \\
\hline TT + TC vs. CC & fixed & 38.31 \\
\hline TT vs. TC + CC & random & 53.74 \\
\hline
\end{tabular}

\section{Ethnicity}

Caucasian

Caucasian

Tvs. C fixed 35.99

\begin{tabular}{l|l|l|l|l}
\hline TT vs. CC & fixed & 50.78 \\
\hline
\end{tabular}

TC vs. CC fixed

0.00

TT + TC vs. CC

Asian

\begin{tabular}{|c|c|c|c|c|c|c|}
\hline TT vs. TC + CC & fixed & 48.03 & 0.123 & 0.767 & $0.423-1.390$ & -0.875 \\
\hline T vs. C & fixed & 35.19 & 0.214 & 1.472 & $1.174-1.844$ & 3.358 \\
\hline TT vs. CC & fixed & 48.33 & 0.144 & 2.063 & $1.247-3.414$ & 2.818 \\
\hline TC vs. CC & fixed & 0.00 & 0.533 & 1.544 & $1.110-2.148$ & 2.577 \\
\hline TT + TC vs. CC & fixed & 0.00 & 0.383 & 1.646 & $1.207-2.244$ & 3.148 \\
\hline TT vs. TC + CC & fixed & 45.83 & 0.158 & 1.568 & $0.995-2.470$ & 1.940 \\
\hline
\end{tabular}

14.52

0.036

1.194

\begin{tabular}{|l|l|}
0.014 & 1.348 \\
\hline
\end{tabular}

$0.416 \quad 1.276$

\begin{tabular}{|l|l|}
0.137 & 1.268 \\
\hline
\end{tabular}

$0.043 \quad 1.171$

0.940-1.518

$0.689-2.641$

1.047-1.555

1.050-1.532

0.669-2.051

1.453

0.872

0.145

1.000

0.909

2.410

0.383

1.000

0.938

2.410

0.016

1.000

0.942

2.470

0.014

1.000

0.908

0.580

0.763

0.867

\begin{tabular}{|l|l|l|l|l|l|l|}
\hline 0.196 & 1.017 & $0.831-1.244$ & 0.163 & 0.871 & 1.000 & 0.812 \\
\hline 0.107 & 0.766 & $0.417-1.406$ & -0.861 & 0.389 & 0.734 & 0.491 \\
\hline 0.423 & 1.146 & $0.895-1.468$ & 1.081 & 0.280 & 0.308 & 0.170 \\
\hline 0.320 & 1.088 & $0.858-1.380$ & 0.699 & 0.489 & 0.308 & 0.431 \\
\hline 0.123 & 0.767 & $0.423-1.390$ & -0.875 & 0.382 & 0.308 & 0.409 \\
\hline 0.214 & 1.472 & $1.174-1.844$ & 3.358 & 0.001 & 1.000 & 0.882 \\
\hline 0.144 & 2.063 & $1.247-3.414$ & 2.818 & 0.005 & 1.000 & 0.722 \\
\hline 0.533 & 1.544 & $1.110-2.148$ & 2.577 & 0.010 & 0.296 & 0.257 \\
\hline 0.383 & 1.646 & $1.207-2.244$ & 3.148 & 0.002 & 1.000 & 0.519 \\
\hline 0.158 & 1.568 & $0.995-2.470$ & 1.940 & 0.052 & 1.000 & 0.890 \\
\hline
\end{tabular}




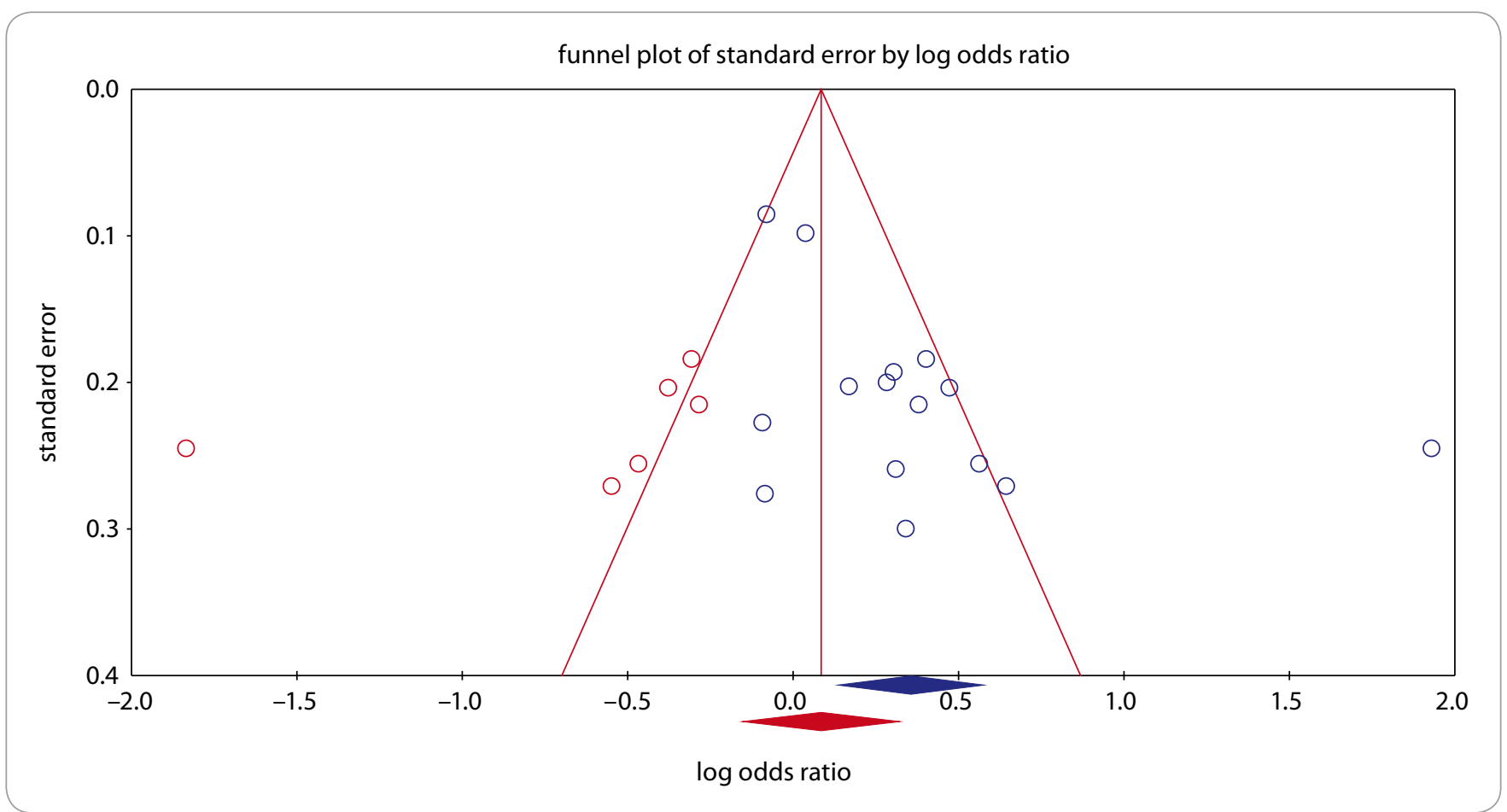

Fig. 3. Funnel plot for publication bias in the meta-analysis of NQO1 rs 1800566 polymorphism with bladder cancer under dominant model (TT + TC vs. CC).

heterozygote (TC vs. CC: OR 1.389, 95\% $\mathrm{Cl} 1.111-1.738, \mathrm{P}=0.004)$, and dominant (TT + TC vs. CC: OR $1.428,95 \% \mathrm{Cl}$ $1.145-1.782, \mathrm{P}=0.002)$. Stratified analysis by ethnicity revealed that there was a significant association between NQO1 rs1800566 polymorphism and bladder cancer among Caucasians using all five genetic models, i.e., allele (T vs. C: OR $1.421,95 \% \mathrm{Cl} 1.249-1.616, \mathrm{P} \leq 0.001)$, homozygote (TT vs. CC: OR $1.890,95 \% \mathrm{Cl}$ 1.445-2.472, $\mathrm{P} \leq 0.001)$, heterozygote (TC vs. CC: OR $1.469,95 \%$ Cl 1.208-1.787, $\mathrm{P} \leq 0.001)$, dominant (TT + TC vs. CC: OR $1.583,95 \%$ Cl 1.318-1.901, $\mathrm{P} \leq 0.001)$, and recessive (TT vs. TC + CC: OR 1.498, 95\% Cl 1.183-1.897, $\mathrm{P}=0.001$ ), but not in Asian population.

\section{Prostate Cancer}

The summary of the meta-analysis of the association of NQO1 rs 1800566 polymorphism with prostate cancer is shown in Tab. 2. Overall, pooled ORs showed that there was a significant association between NQO1 rs1800566 polymorphism and prostate cancer risk under two genetic models, i.e., heterozygote (TC vs. CC: OR 1.276, 95\% Cl 1.047-1.555, $\mathrm{P}=0.016)$, and dominant (TT $+\mathrm{TC}$ vs. CC:
OR $1.268,95 \% \mathrm{Cl} 1.050-1.532, \mathrm{P}=0.014)$ (Fig. 2B). Stratified analysis by ethnicity revealed that there was a significant association between NQO1 rs1800566 polymorphism and prostate cancer among Asians under all four genetic models, i.e., allele (T vs. C: OR $1.472,95 \% \mathrm{Cl}$ 1.174-1.844, $\mathrm{P}=0.001$ ), homozygote (TT vs. CC: OR $2.063,95 \%$ Cl 1.247-3.414, $\mathrm{P}=0.005$ ), heterozygote (TC vs. CC: OR $1.544,95 \% \mathrm{Cl} 1.110-2.148, \mathrm{P}=0.010)$, dominant (TT + TC vs. CC: OR 1.646, 95\% $\mathrm{Cl} 1.207-2.244, \mathrm{P}=0.002)$, but not under Caucasians.

\section{Heterogeneity test and sensitivity analyses}

There was a statistically significant heterogeneity for both bladder cancer and prostate cancer in the overall analysis. Thus, we performed subgroup analyses by ethnicity and HWE status to explain the potential source of heterogeneity. As shown in Tab. 2, most heterogeneity disappeared in the subgroup analysis by ethnicity among Asians and Caucasians, indicating that ethnicity might be the major source of heterogeneity in this meta-analysis. Moreover, we performed a sensitivity analysis to assess the influ- ence of each study on pooled results and robustness of our results by sequential omission of each eligible study. However, the pooled results showed that the significance of the OR was not affected by any single study. Then, sensitivity analysis was conducted by excluding those studies departure from the HWE. Therefore, the sensitivity analysis suggested that the current meta-analysis were relatively consistent even when a single study or some studies were excluded.

\section{Publication bias}

Publication bias was assessed with Begg's funnel plots and Egger's test (Tab. 2). The shapes of the funnel plots and Egger's test did not show any evidence of publication bias in the overall and subgroup analysis by ethnicity for prostate cancer. However, the results of Begg's funnel plots and Egger's regression test suggested evidence of publication bias for bladder cancer in overall under two genetic models, i.e., allele $\left(P_{\text {Beggs }}=0.276 ; P_{\text {Eggers }}=0.014\right)$ and dominate $\left(P_{\text {Beggs }}=0.198 ; P_{\text {Eggers }}=0.023 ;\right.$ Fig. 3$)$, and by subgroup analysis among Asians under the allele model $\left(P_{\text {Beggs }}=0.259\right.$; 
$\left.P_{\text {Eggers }}=0.027\right)$. Thus, to adjust these biases, we used a trim-and-fill method developed by Duval and Tweedie. However, after trimming, we obtained similar results, indicating that the results were statistically reliable.

\section{Discussion}

In this meta-analysis, a total of 22 casecontrol studies including 15 studies on bladder cancer and 7 on prostate cancer were selected to provide the most comprehensive assessment of the association of NQO1 rs1800566 polymorphism with bladder cancer and prostate cancer risk. The current meta-analysis results showed that the NQO1 rs1800566 polymorphism is significantly associated with bladder and prostate cancers. Moreover, stratified analysis by ethnicity showed that the NQO1 rs1800566 polymorphism was significantly associated with an increased risk of bladder and prostate cancers in Caucasians and Asians, respectively. In view of the complex effect of genetic variations on tumorigenesis, the lack of an increased risk of bladder and prostate cancers with NQO1 rs1800566 polymorphism in other populations might be attributed to genetic backgrounds and environmental factors of those populations.

The current meta-analysis results are inconsistence with previous meta-analysis investigating the association between NQO1 rs1800566 polymorphism in the prostate cancer risk. In 2014, Zhang et al., in a meta-analysis of 6 case-control studies with 717 cases and 1,794 controls, failed to show a significant association between the NQO1 rs1800566 polymorphism and prostate cancer risk in overall population. However, similarly to our results, they reported that the NQO1 rs1800566 polymorphism might be a risk factor for development of prostate cancer in Asians [35]. However, Zhang et al., in meta-analysis of urinary system cancer including 5 casecontrols on prostate cancer found that the NQO1 rs1800566 polymorphism conferred genetic susceptibility to urinary system cancer including bladder cancer, prostate cancer, and renal cell carcinoma [11]. Moreover, our results are inconsistent with previous meta- analysis only focused on the role of NQO1 rs1800566 polymorphism in the bladder cancer risk [36,37]. Recently, Wang et al. performed a meta-analysis on effects of NQO1 rs1800566 polymorphism and smoking as an environment-related factor on bladder cancer susceptibility. Their meta-analysis included seven case-control studies with 1,341 cases and 1,346 controls, and showed that the NQO1 rs1800566 polymorphism in smokers significantly increased risk of bladder cancer compared with non-smokers [12]. However, their estimates were based on crude pooled ORs, not adjusted OR values, such as ethnicity, which might be the cause of inaccurate results. Similarly, in 2014, Goerlitz et al., in a case-control study of 902 cases with bladder cancer and 804 population-based healthy subjects in Egypt found that the NQO1 rs1800566 polymorphism plays an important role in the susceptibility to bladder cancer by modulating the effects of known contributing factors, such as smoking and also schistosoma haematobium infection [17].

Meta-analysis is an ideal tool to identify genetic association [38]. However, between-study heterogeneity can distort the results of a meta-analysis $[39,40]$. Several factors, such as diversity in study design, sample size, ethnicity, source of controls, genotyping method, and deviation from HWE might contribute to results heterogeneity $[41,42]$. To identify possible factors that contributed to high heterogeneity, we performed subgroup analysis by ethnicity. The overall heterogeneity no longer existed in subgroup analysis, thus we hypothesized that ethnicity was the main source of between-study heterogeneity in this meta-analysis.

Some limitations in this meta-analysis must be addressed. First, the small sample size for prostate cancer was the major defect in this meta-analysis. Thus, welldesigned studies with large sample size are needed to further investigate the association between NQO1 rs1800566 polymorphism and prostate cancer risk. Second, we mostly focused on studies published in English and Chinese, which might have led to publication bias. Third, the study populations were dominantly Caucasian and Asian. The subgroup meta-analysis for ethnicity had little or no information for other ethnic groups, such as Africans and mixed populations. Forth, several important confounding factors, such as age, gender (for bladder cancer), drinking, smoking, and disease stages were not considered for stratified analysis because relevant data were insufficient in the selected studies. Finally, bladder and prostate cancers are mainly caused by gene-gene and geneenvironment interactions. However, no appropriate information was available for further analysis and data sorting. Therefore, further large-scale studies in different populations with more detailed data, with different environmental background are required to validate gene-gene and gene-environment interactions on NQO1 rs 1800566 polymorphism with risk of bladder and prostate cancers.

In summary, the results of meta-analysis suggested that the NQO1 rs1800566 polymorphism was significantly associated with an increased risk of bladder and prostate cancers. Moreover, NQO1 rs1800566 polymorphism was significantly associated with risk of bladder cancer and prostate cancer in Caucasians and Asians, respectively. However, well-designed and large studies are needed to further investigate the association of these polymorphisms with breast cancer susceptibility.

\section{References}

1. Wong MC, Fung FD, Leung $C$ et al. The global epidemiology of bladder cancer: a joinpoint regression analysis of its incidence and mortality trends and projection. Sci Rep 2018; 8(1): 1129. doi: 10.1038/s41598-018-19199-z. 2. Gunlusoy B, Ceylan Y, Degirmenci T et al. The potential effect of age on the natural behavior of bladder cancer: Does urothelial cell carcinoma progress differently in various age groups? Kaohsiung J Med Sci 2016; 32(5): 261-266. doi: 10.1016/j.kjms.2016.03.002.

3. Sanli O, Dobruch J, Knowles MA et al. Bladder cancer. Nat Rev Dis Primers 2017; 3: 17022. doi: 10.1038/nrdp.2017.22. 4. Bashir MN. Epidemiology of prostate cancer. Asian Pac J Cancer Prev 2015; 16(13): 5137-5141. doi: 10.7314/apjcp.2015.16.13.5137.

5. Abedinzadeh M, Zare-Shehneh M, Neamatzadeh $\mathrm{H}$ et al. Association between MTHFR C677T polymorphism and risk of prostate cancer: evidence from 22 studies with 10,832 cases and 11,993 controls. Asian Pac J Cancer Prev 2015; 16(11): 4525-4530. doi: 10.7314/apjcp.2015.16.11.4525.

6. Madersbacher S, Alcaraz A, Emberton M et al. The influence of family history on prostate cancer risk: impli- 
cations for clinical management. BJU Int 2011; 107(5): 716-721. doi: 10.1111/j.1464-410X.2010.10024.x.

7. Dudek AM, Grotenhuis AJ, Vermeulen SH et al. Urinary bladder cancer susceptibility markers. What do we know about functional mechanisms? Int J Mol Sci 2013; 14(6): 12346-12366. doi: 10.3390/ijms140612346.

8. Huang ZM, Chen HA, Chiang YT et al. Association of polymorphisms in $\mathrm{NNOS}$ and NQO1 with bladder cancer risk in cigarette smokers. J Chin Med Assoc 2014; 77(2): 83-88. doi: 10.1016/j.jcma.2013.10.005.

9. Ergen HA, Gormus $U$, Narter $F$ et al. Investigation of $\mathrm{NAD}(\mathrm{P}) \mathrm{H}$ : quinone oxidoreductase 1 (NQO1) C609T polymorphism in prostate cancer. Anticancer Res 2007; 27(6B): 4107-4110.

10. Jaiswal AK. Human NAD(P)H: quinone oxidoreductase (NQO1) gene structure and induction by dioxin. Biochemistry 1991; 30(44): 10647-10653. doi: 10.1021/bi00108a007

11. Zhang Y, Yang D, Zhu JH et al. The association between NQO1 Pro187Ser polymorphism and urinary system cancer susceptibility: a meta-analysis of 22 studies. Cancer Invest 2015; 33(2): 39-40. doi: 10.3109/07357907.2014.998836.

12. Wang XC, Wang J, Tao HH et al. Combined effects of NQO1 Pro187Ser or SULT1A1 Arg213His polymorphism and smoking on bladder cancer risk: two meta-analyses. Int J Occup Med Environ Health 2017; 30(5): 791-802. doi: 10.13075/ijomeh.1896.00930

13. Yadav U, Kumar P, Rai V. NQO1 gene C609T polymorphism (dbSNP: rs 1800566) and digestive tract cancer risk: a meta-analysis. Nutr Cancer 2018; 70(4): 557-568. doi: 10.1080/01635581.2018.1460674.

14. Ding R, Lin S, Chen D. Association of NQO1 rs 1800566 polymorphism and the risk of colorectal cancer: a metaanalysis. Int J Colorectal Dis 2012; 27(7): 885-892. doi: 10.1007/s00384-011-1396-0

15. Schulz WA, Eickelmann $\mathrm{P}$, Sies H. Free radicals in toxicology: redox cycling and $\mathrm{NAD}(\mathrm{P}) \mathrm{H}$ : quinone oxidoreductase. Arch Toxicol Suppl 1996; 18: 217-222. doi 10.1007/978-3-642-61105-6_22.

16. Pandith AA, Khan NP, Shah ZA et al. Association of bladder cancer risk with an $\mathrm{NAD}(\mathrm{P}) \mathrm{H}$ : quinone oxidoreductase polymorphism in an ethnic Kashmiri population. Biochem Genet 2011; 49(7-8): 417-426. doi: 10.1007/s10528-011-9418-8

17. Goerlitz D, Amr S, Dash C et al. Genetic polymorphisms in NQO1 and SOD2: interactions with smoking, schistosoma infection, and bladder cancer risk in Egypt. Urol Oncol 2014; 32(1): 15-20. doi: 10.1016/j.urolonc.2013.06.016

18. Mandal RK, Nissar K, Mittal RD. Genetic variants in metabolizing genes NQO1, NQO2, MTHFR and risk of prostate cancer: a study from North India. Mol Biol Rep 2012; 39(12): 11145-11152. doi: 10.1007/s11033-0122023-z
19. Wang $\mathrm{YH}$, Lee $\mathrm{YH}$, Tseng PT et al. Human NAD(P)H: quinone oxidoreductase 1 (NQO1) and sulfotransferase 1A1 (SULT1A1) polymorphisms and urothelial cancer risk in Taiwan. J Cancer Res Clin Oncol 2007; 134(2): 203-209. doi: 10.1007/s00432-007-0271-4

20. Fu J, Chen B. Relationship between genetic polymorphisms of CYP1A1, NQO1 and EPHX1 and susceptibility to bladder cancer. Chin J Cancer Prev Treat 2013; 20(1): 10-14.

21. Park SJ, Zhao H, Spitz MR et al. An association between NOO1 genetic polymorphism and risk of blad der cancer. Mutat Res 2003; 536(1-2): 131-137. doi 10.1016/s1383-5718(03)00041-x

22. Choi JY, Lee KM, Cho SH et al. CYP2E1 and NQO1 genotypes, smoking and bladder cancer. Phar macogenetics 2003; 13(6): 349-355. doi: 10.1097/01. fpc.0000054096.48725.25

23. Sanyal S, Festa F, Sakano S et al. Polymorphisms in DNA repair and metabolic genes in bladder cancer. Carcinogenesis 2004; 25(5): 729-734. doi: 10.1093/carcin/bgh058.

24. Hung RJ, Boffetta P, Brennan P et al. Genetic polymorphisms of MPO, COMT, MnSOD, NQO1, interaction with environmental exposures and bladder cancer risk Carcinogenesis 2004; 25(6): 973-978. doi: 10.1093/carcin/bgh080.

25. Moore LE, Wiencke JK, Bates MN et al. Investigation of genetic polymorphisms and smoking in a bladder cance case-control study in Argentina. Cancer Lett 2004; 211 (2): 199-207. doi: 10.1016/j.canlet.2004.04.011.

26. Terry PD, Umbach DM, Taylor JA. No association between SOD2 or NQO1 genotypes and risk of bladde cancer. Cancer Epidemiol Biomarkers Prev 2005; 14(3) 753-754. doi: 10.1158/1055-9965.EPI-04-0574.

27. Broberg K, Björk J, Paulsson Ket al. Constitutional short telomeres are strong genetic susceptibility markers for bladder cancer. Carcinogenesis 2005; 26(7): 1263-1271. doi: 10.1093/carcin/bgi063.

28. Figueroa JD, Malats N, García-Closas M et al. Bladder cancer risk and genetic variation in AKR1C3 and other metabolizing genes. Carcinogenesis 2008; 29(10): 1955-1962. doi: 10.1093/carcin/bgn 163

29. Steiner M, Hillenbrand M, Borkowsi M et al. 609 C-T polymorphism in $\mathrm{NAD}(\mathrm{P}) \mathrm{H}$ : quinone oxidoreductase gene in patients with prostatic adenocarcinoma or be nign prostatic hyperplasia. Cancer Lett 1999; 135(1): 67-71. doi: 10.1016/s0304-3835(98)00269-9.

30. Hamajima N, Matsuo K, Iwata $\mathrm{H}$ et al. NAD(P)H: quinone oxidoreductase 1 (NQO1) C609T polymorphism and the risk of eight cancers for Japanese. Int J Clin Onco 2002: 7(2): 103-108 doi: 10.1007/s101470200013.

31. Ergen HA, Gormus U, Narter F et al. Investigation of $\mathrm{NAD}(\mathrm{P}) \mathrm{H}$ : quinone oxidoreductase 1 (NQO1) C609T polymorphism in prostate cancer. Anticancer Res 2007; 27(6B): 4107-4110.
32. Stoehr CG, Nolte E, Wach S et al. NAD(P)H: quinone oxidoreductase 1 (NQO1) P187S polymorphism and prostate cancer risk in caucasians. Int J Mol Sci 2012; 13(9): 10959-10969. doi: 10.3390/ijms130910959.

33. Jing-Xian Z, Li-Ling L, Ya-Wen W. NQO1 C609T gene polymorphism associated with an increased risk of prostate cancer cognitive dysfunction. Chin J Behav Med and Brain Sci 2011; 20(8): 698-700.

34. Steinbrecher A, Rohrmann S, Timofeeva M et al. Dietary glucosinolate intake, polymorphisms in selected biotransformation enzymes, and risk of prostate cancer. Cancer Epidemiol Biomarkers Prev 2010; 19(1): 135-143. doi: 10.1158/1055-9965.EPI-09-0660.

35. Zhang $\mathrm{DH}$, Zhang $\mathrm{Q}$, Zheng $M$ et al. Effect of NQO1 C609T polymorphism on prostate cancer risk: a meta-analysis. Onco Targets Ther 2014; 7: 907. doi: 10.2147/OTT.S62046.

36. Yang S, Jin T, Su HX et al. The association between NQO1 Pro187Ser polymorphism and bladder cancer susceptibility: a meta-analysis of 15 studies. Plos One 2015; 10(1): e0116500. doi: 10.1371/journal.pone.0116500.

37. Gong $M$, Yi Q, Wang W. Association between NQO1 C609T polymorphism and bladder cancer susceptibility: a systemic review and meta-analysis. Tumor Biol 2013; 34(5): 2551-2556. doi: 10.1007/s13277-013-0799-7. 38. Jafari Nedooshan J, Kargar S, Neamatzadeh H et al. Lack of association of the fat mass and obesity associated (FTO) gene rs9939609 polymorphism with breast cancer risk: a systematic review and meta-analysis based on case - control studies. Asian Pac J Cancer Prev 2017; 18(4): 1031-1037. doi: 10.22034/APJCP.2017.18.4.1031.

39. Aslebahar F, Neamatzadeh $\mathrm{H}$, Meibodi B et al. Association of tumor necrosis factor- $a$ (TNF-a) -308G $>$ A and $-238 \mathrm{G}>\mathrm{A}$ polymorphisms with recurrent pregnancy loss risk: a meta-analysis. Int J Fertil Steril 2019; 12(4): 284-292. doi: 10.22074/ijfs.2019.5454

40. Kamali M, Hamadani S, Neamatzadeh H et al. Association of XRCC2 rs3218536 polymorphism with susceptibility of breast and ovarian cancer: a systematic review and meta-analysis. Asian Pac J Cancer Prev 2017; 18(7): 1743-1749. doi: 10.22034/APJCP.2017.18.7.1743.

41. Namazi A, Forat-Yazdi M, Jafari M et al. Association of interleukin-10 -1082 A/G (rs1800896) polymorphism with susceptibility to gastric cancer: meta-analysis of 6,101 cases and 8,557 controls. Arq Gastroenterol 2018; 55(1): 33-40. doi: 10.1590/s0004-2803.201800000-18. 42. Moghimi M, Kargar S, Jafari MA et al. Angiotensin converting enzyme insertion/deletion polymorphism is associated with breast cancer risk: a meta-analysis. Asian Pac J Cancer Prev 2018; 19(11): 3225-3231. doi: 10.31557/ APJCP.2018.19.11.3225.

43. Moher D, Liberati A, Tetzlaff J et al. Preferred reporting items for systematic reviews and meta-analyses: the PRISMA statement. PLoS Med 2009; 6(7): e1000097. doi: 10.1371/journal.pmed.1000097. 